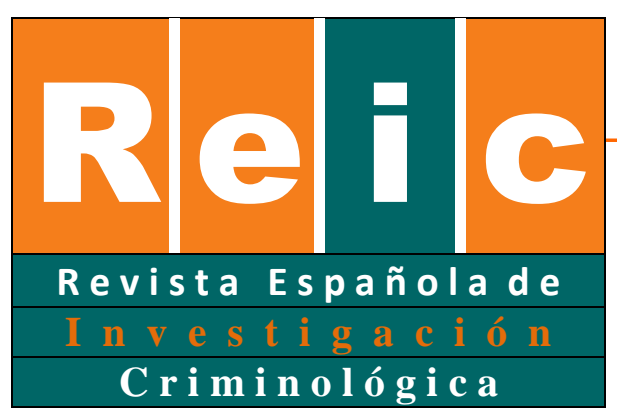

Giménez-Salinas et al.

\title{
Estructuras y liderazgo en cuatro redes españolas dedicadas al tráfico de drogas
}

Manuscrito recibido el 4 de mayo del 2012/ Publicado el 1 de agosto de 2012.

\author{
Andrea Giménez-Salinas Framis, Hugo Román Ojea, Luisa Nieto \\ Corominas, Antonio José Martínez Antón, Sonia Fernández Regadera ${ }^{1}$ \\ Instituto de Ciencias Forenses y de la Seguridad \\ Universidad Autónoma de Madrid
}

\section{RESUMEN}

Este artículo tiene como objetivo estudiar, desde la perspectiva del análisis de redes sociales, la estructura, las personas más relevantes y las posiciones jerárquicas de los miembros de cuatro grupos involucrados en el tráfico de cocaína de nivel medio (mayoristas). Con este fin, se ha recabado información de los archivos policiales sobre cuatro operaciones criminales, a través de un cuestionario de 76 variables, divididas en cuatro áreas principales: las características de la organización, el mercado ilícito, actividades instrumentales o transversales y el perfil de los miembros del grupo. También se ha recogido información sobre contactos telefónicos y reuniones registradas en la investigación policial, con el fin de analizar la organización desde la perspectiva del análisis de redes sociales. Las cuatro redes han sido examinadas teniendo en cuenta tres aspectos: la estructura, los actores centrales e intermediarios y sus posiciones jerárquicas. Los resultados muestran diferencias con los enfoques tradicionales jerárquicos y avanzan posibles aplicaciones para la investigación y la inteligencia criminal.

Palabras clave: análisis de redes sociales, estructura del crimen organizado, redes de tráfico de drogas.

\footnotetext{
${ }^{1}$ El presente trabajo ha sido realizado en seno del Instituto de Ciencias Forenses y de la Seguridad por un grupo de trabajo formado por colaboradores de dicho instituto que a la vez son alumnos de posgrado. Sin embargo, este trabajo no hubiera sido posible sin la ayuda inestimable de la Guardia Civil (Unidad Técnica de Policía Judicial). Agradecemos la colaboración prestada pero no podemos responsabilizarles del contenido y la interpretación de los datos que corresponde únicamente a los autores del trabajo. Queremos también agradecer los comentarios y recomendaciones realizados por el editor de la revista y los evaluadores anónimos. Agradecemos su atenta lectura y sus comentarios que, sin duda, han enriquecido el artículo y han mejorado determinados argumentos.
} 


\begin{abstract}
The aim of the paper is to present the findings of a research which targets the application of social network analysis (SNA) perspective to data coming from police investigations about four middle level drug trafficking organizations. For that purpose, we have collected information from police files of 4 investigations of criminal organizations by means of a questionnaire of 76 variables divided in four main areas: features of the organization, illicit market, instrumental or transversal activities and profile of members. Additionally, we have gathered information about wiretappings and meetings registered by the criminal investigation with the intention to analyze the organization under SNA perspective. The four networks have been analyzed taking into account three dimensions: a) structure and inside roles; b) powerful members; c) hierarchical positions. Results show up differences between networks and advantages of SNA perspective in comparison with more hierarchical approaches and further applications for investigation and intelligence purposes.
\end{abstract}

Key words: social network analysis, organized crime structures, drug trafficking networks

\title{
1. Introducción
}

La criminología ha dedicado tradicionalmente sus mayores esfuerzos al estudio de la delincuencia de mayor prevalencia: la delincuencia común o juvenil. En referencia a los fenómenos grupales, también las bandas juveniles han ocupado un lugar prioritario en la literatura criminológica, quedando el estudio de las organizaciones formadas por adultos, relegado a un segundo plano. Dichas razones explican que la delincuencia adulta y relacionada con la criminalidad organizada haya permanecido en la sombra de la curiosidad científica, y que las pocas aportaciones teóricas existentes resulten manifiestamente insuficientes (Kleemans y de Poot, 2008; Morselli, 2009). Por otro lado, el origen de las disciplinas que han mostrado curiosidad por este fenómeno así como las dificultades que entraña la investigación en este campo, explican su abordaje más teórico que empírico. Por todo ello y, a pesar de los pasos avanzados en los últimos años, todavía quedan muchos interrogantes sin respuesta: ¿cómo se organizan los delincuentes adultos en los grupos criminales?, ¿cómo se distribuyen los roles en el interior de los grupos? ¿Cuáles son las estructuras más comunes en los mercados ilícitos tradicionales del crimen organizado? ¿Cómo responden y se recomponen los 
grupos después de la detención policial? ¿Qué elementos favorecen la resiliencia ${ }^{2}$ de las organizaciones?

Este artículo describe los resultados de un estudio sobre cuatro grupos criminales intermediarios o de nivel medio, según las tipologías sugeridas por la literatura científica. Esta categoría se consagra a aquellos grupos que sirven de enlace entre el proveedor o importador de la droga procedente de los países de origen y el distribuidor local, ejerciendo una actividad propia de los mayoristas o principales proveedores locales (middle level groups, en inglés). Todos los grupos estudiados operan en el mercado ilícito de la cocaína y uno de ellos también se dedica al tráfico de heroína. Asimismo, los cuatro han sido investigados por la policía entre 2007 y 2009.

El objetivo de la investigación consiste en comparar la información proveniente de la investigación criminal llevada a cabo por la policía en las cuatro operaciones policiales y la información procedente del análisis de redes sociales (en adelante, ARS) realizado a partir de los contactos telefónicos ${ }^{3}$ detectados entre los miembros de los grupos criminales. Dicho objetivo tiene como finalidad principal conocer las ventajas del ARS y, de forma secundaria, verificar si estamos ante un perfil organizacional más propio de organizaciones criminales complejas (jerárquicamente estructuradas) o de redes criminales más flexibles. Para ello, abordaremos tres dimensiones: a) la estructura de los grupos; b) los actores principales de la organización y c) las posiciones jerárquicas dentro de la red.

\footnotetext{
${ }^{2}$ El concepto de resiliencia fue utilizado en primer lugar por físicos y matemáticos para describir la habilidad de ciertos materiales para volver a su forma original después de un desplazamiento (Norris y otros, 2008). Este concepto ha sido luego importado a otras disciplinas sociales para estudiar la estabilidad de los sistemas o individuos. Llevado el concepto al terreno de las organizaciones, la resiliencia sería la habilidad de un grupo de absorber la disrupción o de reorganizarse después de un cambio para volver a las mismas funciones, estructura e identidad (Walker y otros, 2004:6; Ayling, 2009 y Horne, 1997).

3 Los contactos telefónicos registrados en la operación policial, así como las reuniones realizadas entre los miembros de la organización se han tenido en cuenta como datos de contacto para proceder al análisis de redes sociales. De los contactos, únicamente se ha registrado la cantidad y dirección de los mismos, evitando la identificación de los sujetos y preservando el anonimato.
} 


\section{Marco teórico}

\subsection{Organizaciones criminales o redes ilegales}

El interior de las organizaciones criminales constituye una cuestión tradicional de estudio en el marco de la delincuencia organizada y un tema en auge en la literatura criminológica actual. Hace unas décadas y gracias a la aportación de Cressey, en su obra The theft of the nation, se empezaron a abordar las estructuras de las grandes mafias. Cressey, al descubrirnos la estructura y entresijos del principal sindicato americano del crimen (Cosa Nostra), nos propuso un prototipo de organización criminal al más puro estilo weberiano que se ha denominado modelo burocrático. Este enfoque organizacional se caracteriza por los siguientes elementos: una jerarquía centralizada, una clara división de funciones, la asignación de roles en función de las habilidades personales y un código normativo formalmente establecido para mantener la cohesión interna. Empero, este enfoque resulta útil para describir con precisión las estructuras de tipo mafioso pero adolece de serias limitaciones cuando lo aplicamos a las organizaciones criminales que desarrollan su actividad en la actualidad. De hecho, ya muchos de los autores posteriores a Cressey mostraron su disconformidad con el modelo (Reuter, 1985) por considerarlo inadecuado para la comprensión de organizaciones tradicionales. En la actualidad, debido a la globalización, las nuevas tecnologías de comunicación y el aumento del control policial, el modelo burocrático explica de forma limitada la tendencia actual de los grupos a participar de estructuras mucho más flexibles (Williams, 2001; Paoli, 2002; Hobbs, 2001 y Morselli, 2009).

Recientemente, el estudio del esqueleto (usando la terminología acuñada por Cressey) de las organizaciones ha dado un importante paso hacia delante con la introducción del ARS. Este método constituye una forma alternativa de adentrarse en el interior de las organizaciones criminales (en tanto sistema social o red de relaciones), a través del estudio de las relaciones sociales que se generan entre los miembros de una organización o red. El ARS tiene como objetivo genérico, el estudio de los nodos o unidades de la red (sujeto, grupos, etc.) y los vínculos que se producen entre ellos. En este sentido, la aplicación de esta metodología al estudio de las organizaciones criminales nos ayuda a entender, con mayor precisión, las dinámicas de 
comunicación y relación que se producen entre los miembros de la organización, sin partir de un organigrama o esquema preconcebido (McIllman,1999 y Morselli, 2009).

Esta metodología ha sido muy utilizada para el estudio de redes terroristas que se integran dentro del término genérico covert networks o dark networks. Dentro de este género se encuentran los grupos sociales que se desarrollan y mantienen en la clandestinidad y que, por razones de seguridad, su actividad debe permanecer en secreto (grupos terroristas u organizaciones criminales). Este tipo de redes son difíciles de abordar pero el ARS ha revelado una metodología capaz de analizar e interpretar la estructura y dinámicas de dichas redes clandestinas sin necesidad de contar con excesiva información, únicamente a partir de los contactos que se producen entre los miembros. En los últimos años, numerosos estudios han estudiado diversas dimensiones de las redes clandestinas utilizando el ARS: su funcionamiento interno (Morselli, 2009), sus características internas (Canter, 2004), la identificación de sujetos claves y sus puntos débiles (Borgatti, 2006), la predicción de las dinámicas internas y el comportamiento de sus miembros (Monge y Contractor, 2003) o la forma de desestabilizarlas y reducir su capacidad (Carley, Lee y Krackhardt, 2001; Coutu, 2002; Sheffi, 2005).

El equipo de trabajo que presenta este estudio considera el ARS como un método complementario y no exclusivo de análisis que mejora la comprensión de las organizaciones $^{4}$. Asimismo, partimos de una perspectiva conservadora que concibe las organizaciones criminales y las redes ilegales como diferentes formas organizacionales que constituyen extremos de un continuo (desde estructuras más complejas y rígidas a más flexibles) ${ }^{5}$. A continuación presentamos una tabla que resume las señas de

\footnotetext{
${ }^{4}$ Ciertos sectores académicos conciben el análisis de redes sociales como una única herramienta capaz de comprender las relaciones internas y la estructura de los grupos. Nosotros consideramos que el ARS aporta información adicional a la que proporciona la investigación tradicional de los grupos criminales. Su uso enriquece la investigación puesto que nos ayuda a identificar rápidamente las dinámicas de poder y la estructura interna de los grupos.

${ }_{5}$ Dos enfoques no necesariamente compatibles coexisten en el abordaje del concepto de red en las organizaciones criminales. El primer enfoque entiende la red como una forma de organización inmersa en un continuo cuyos extremos van desde grupos más jerarquizados y complejos a grupos más horizontales, flexibles y reticulares. Este último tipo de estructuras es especialmente prevalente en el crimen organizado de tipo empresarial (Von Lampe, 2008). El segundo enfoque considera la red como un concepto que transciende las formas organizacionales y constituye el principal objeto de estudio (Morselli,

2009).
} 
identidad de cada una de estas formas organizacionales. Dicho resumen nos ayudará también a interpretar si las organizaciones que han sido objeto de análisis en este artículo se consideran más cercanas a la categoría de organización criminal o de red ilegal.

Tabla 1. Características de las organizaciones criminales versus las redes ilegales

\begin{tabular}{|c|c|c|}
\hline & Organizaciones criminales & Redes ilegales \\
\hline Estructura & Jerárquica & Horizontal \\
\hline Division roles & Division de tareas, especialización & $\begin{array}{l}\text { Roles operacionales } \\
\text { intercambiables }\end{array}$ \\
\hline Movilidad interna & Miembros fijos y permanentes & Miembros no fijos ni permanentes \\
\hline Promoción interna & $\begin{array}{l}\text { Sistemas promocionales y } \\
\text { procedimientos de selección de } \\
\text { candidatos por méritos o } \\
\text { habilidades }\end{array}$ & $\begin{array}{l}\text { Reclutamiento y selección basada } \\
\text { oportunidades y lazos sociales: } \\
\text { antiguos negocios, etnicidad o } \\
\text { familia, contactos, asignaciones } \\
\text { concretas, etc. (Ianni, 1972) }\end{array}$ \\
\hline Normas & Normas formales y secretas & Normas flexibes e inestables \\
\hline Comunicación & $\begin{array}{l}\text { Comunicación formal (se comparte } \\
\text { mucha información) }\end{array}$ & $\begin{array}{l}\text { Comunicación directa e individual } \\
\text { (se comparte poca información) }\end{array}$ \\
\hline Liderazgo & $\begin{array}{l}\text { Liderazgo basado en la posición } \\
\text { jerárquica }\end{array}$ & $\begin{array}{l}\text { Liderazgo basado en la posesión de } \\
\text { contactos y recursos } \\
\text { (intermediación) }\end{array}$ \\
\hline Protección & $\begin{array}{l}\text { Relación global y Protección global } \\
\text { de la red }\end{array}$ & $\begin{array}{l}\text { Protección individual y relación } \\
\text { individual }\end{array}$ \\
\hline Resiliencia & Estructuras vulnerables & Estructuras resistentes \\
\hline
\end{tabular}

Fuente: elaboración propia a partir de la revisión de la literatura

Además de las características definitorias relativas a su estructura interna, muchos autores creen que las organizaciones con estructuras reticulares son más resistentes a la identificación y detección policial como resultado de sus ventajas estructurales y jerárquicas (Zhang y Chin, 2003; Zaitch, 2002 y Williams, 1998): número de 
miembros, menor estructura burocrática vertical, gran adaptabilidad a las circunstancias y amenazas externas (Benson y Decker, 2010), conexiones débiles entre los miembros y menor centralidad de la organización (Reuter, 1985).

A continuación haremos una referencia especial a las investigaciones que han estudiado el interior de las organizaciones dedicadas al tráfico de drogas.

\subsection{Organización interna del tráfico de drogas}

El tráfico de drogas es una de las actividades ilícitas con más tradición en el ámbito de la delincuencia organizada, razón que explica que hayan sido objeto de un mayor número de investigaciones. Las organizaciones dedicadas a este tráfico ilícito se dividen en tres niveles. El primer nivel se compone de organizaciones dedicadas a la importación de la droga procedente de los países de origen para luego ser vendida en los lugares de destino o de tránsito (Reuter, 1985 y Reuter y Haaga, 1989). El segundo nivel (medio) lo forman los grupos distribuidores al por mayor o mayoristas (nivel intermedio), quienes se encargan de comprar la droga a los importadores y venderla a los distribuidores o minoristas. El tercer nivel está formado por los grupos distribuidores al por menor o minoristas (Adler, 1985) quienes se encargan de la distribución local de la droga. Buena parte de las investigaciones sobre estas organizaciones, especialmente los estudios etnográficos relacionados con la venta de droga a niveles inferiores, han revelado que los grupos que participan en esta actividad no pueden considerarse organizaciones criminales en su versión tradicional y, menos aún, organizaciones al estilo de las grandes mafias. Los sujetos involucrados, los roles ejercidos, los métodos de adulteración utilizados, los beneficios económicos obtenidos, y sus métodos de protección no son propios de grupos delictivos altamente estructurados. Por otra parte, los traficantes de drogas de bajo nivel suelen formar parte de grupos unidos, principalmente, por vínculos basados en el parentesco, la cultura u otras cualidades donde la confianza garantiza el compromiso más allá del interés particular (Gambetta, 1988).

Varios trabajos han confirmado estos resultados en los primeros niveles del proceso de distribución de drogas (nivel medio o superior) (Adler, 1985; 1992; Reuter 
y Haaga, 1989, Dorn et al. 1992; Natarajan y Belanger, 1998; Desroches, 1999; Natarajan, 2000 y Pearson y Hobbs, 2003) destacando la ausencia de evidencias sobre jerarquías verticales en este nivel de redes de tráfico de drogas. Por otro lado, otros estudios han identificado distintas tipologías de grupos, no sólo teniendo en cuenta su distribución jerárquica (estructurada y no estructurada), sino la organización interna de funciones y actividades. En este sentido, se identificaron cuatro tipos de organizaciones (Natarajan y Belanger, 1998):

a) Corporaciones $\mathrm{u}$ organizaciones estructuradas que, en este caso, se asemejan a organizaciones criminales tradicionales definidas anteriormente.

b) Cooperativas o grupos cuyos miembros aúnan sus recursos materiales y económicos para poder desarrollar el tráfico ilícito prácticamente al mismo nivel.

c) Empresas de tipo familiar cuya cohesión se basa en los vínculos de sangre existentes entre los miembros más relevantes.

d) Freelance o sujetos que individualmente poseen contactos para desarrollar determinadas acciones para distintos grupos.

Finalmente, retomando las conclusiones a las que llegan los diferentes estudios relacionados con las organizaciones de medio y alto nivel, podemos destacar que: a) el mercado de drogas no es administrado por grandes organizaciones altamente estructuradas, sino por pequeños grupos y emprendedores cuyos vínculos son débiles y efímeros (Natarajan y Belanger, 1998; Benson y Decker, 2010 y Zaitch, 2002), b) los miembros se implican en los mercados ilegales debido a las oportunidades que se les ofrecen y por vínculos familiares o étnicos (Morselli, 2005, Kleemans y Van de Bunt, 1999), c) los grupos tienen una escasa y flexible división de tareas donde las funciones operativas son intercambiables (muchos de ellos pueden llevar a cabo múltiples funciones), d) los distribuidores de nivel medio o mayoristas juegan un papel bisagra importante entre los importadores y los minoristas, y desarrollan sus actividades prioritariamente a través de contactos que han cultivado a lo largo del tiempo.

Partiendo de los resultados de los trabajos anteriores, la siguiente parte del artículo se consagra a describir los resultados de los análisis de cuatro grupos de nivel 
medio involucrados en el mercado de cocaína y heroína en España. El análisis de estos grupos nos permitirá comprender con más claridad si responden al concepto de organización criminal tradicional y jerárquica (bien integrado entre los investigadores policiales), o si se ajustan mejor a un perfil de red ilegal. A través del ARS, se comprobará si los análisis bajo esta perspectiva confirman las conclusiones de las investigaciones policiales respecto a la estructura de los grupos y su composición interna.

\section{Fuente de datos y diseño de la investigación}

\subsection{Fuente de datos}

El estudio que se presenta a continuación se ha realizado a través del análisis de los contactos telefónicos y reuniones entre los miembros de las organizaciones criminales, obtenidos de la información disponible de los archivos policiales revisados sobre cuatro operaciones policiales ${ }^{6}$. En ellos, se investigan grupos criminales involucrados en el tráfico de cocaína y heroína, que han desarrollado sus actividades principalmente en Madrid. La información recopilada por la policía sobre cada grupo, fue obtenida a partir de las diligencias ${ }^{7}$ recogidas para cada operación policial. Las cuatro operaciones se resumen a continuación:

a) Operación $\mathbf{M}\left(\mathbf{N}=\mathbf{2 2}^{8}\right)$ : esta operación comenzó en el año 2006 y desmanteló un grupo de ciudadanos colombianos que estaban introduciendo $50 \mathrm{~kg}$ de cocaína en Madrid (España) para después ser adulterada y distribuida. El grupo estaba involucrado en la distribución de cocaína en España, importada desde Colombia a través de Brasil y Uruguay, y estaba compuesto por un grupo típico de traficantes de

\footnotetext{
${ }^{6}$ En cada operación policial se pueden identificar más de un grupo criminal, de hecho, como veremos en los resultados de la investigación, cada operación ha supuesto la desarticulación de varios grupos en distintos niveles.
}

\footnotetext{
${ }^{7}$ Las diligencias y contenido de los atestados en la investigación nos proporcionan una información muy rica sobre la organización criminal y los integrantes de la misma. En concreto, cada atestado incluye la totalidad de las actividades de investigación desarrolladas en proceso de investigación llevado a cabo por los agentes policiales, a saber: entradas y registros, interceptación de telecomunicaciones, incautación de efectos, reconocimiento fotográfico, interrogatorios, seguimientos, etc. En la recogida de datos se respetó el derecho a la intimidad de los sujetos investigados y no se recogieron datos personales identificativos de los sujetos.
}

\footnotetext{
${ }^{8}$ La operación se saldó con la identificación de un número mayor de miembros, sin embargo, los análisis de redes se han realizado sobre los miembros sobre los que se han detectado contactos, por ello, los sujetos identificados en las representaciones gráficas no se corresponden con el número de miembros identificados en la operación policial.
} 
cocaína de nivel medio que actuaba como intermediario entre los importadores de América del Sur y los distribuidores locales en Madrid.

b) Operación J (N=49): En 2009 comenzó una operación detectándose un grupo involucrado en actividades de blanqueo de dinero que había sido investigado en anteriores operaciones policiales. El centro operativo de la organización se ubicaba en la Comunidad de Madrid y desde ahí se blanqueaba el dinero de dos organizaciones dedicadas al tráfico de cocaína que actuaban en la península ibérica. Los traficantes de una de las organizaciones a las que servían habían obtenido ganancias económicas estimadas en 60 millones de euros.

c) Operación K (N=41): En el año 2008 se inició una investigación contra un grupo que se dedicaba a la venta y distribución al por mayor y por menor de cocaína y heroína, en una amplia zona de Madrid. Este grupo se encargaba de la adquisición, manipulación y venta de la droga en un conocido poblado gitano caracterizado por una intensa actividad en la compra-venta de drogas.

d) Operación A (N=11): En 2007 se desmanteló un pequeño grupo compuesto principalmente por miembros pertenecientes a la misma familia y liderado por una mujer. Los miembros de la organización criminal distribuían cocaína en Madrid, proveniente de grupos gallegos, que la introducían inicialmente en España. El grupo también poseía sus propios medios y procedimientos para el blanqueo de dinero.

La investigación que a continuación se presenta se llevó a cabo a partir de la recopilación de información cualitativa y cuantitativa procedente de archivos policiales con dos finalidades: en primer lugar, recabar información de los expedientes para completar una hoja de recogida de datos que permitía obtener información sobre los siguientes aspectos: las características del grupo organizado, los negocios ilegales realizados, actividades instrumentales y el perfil de los miembros del grupo ${ }^{9}$.

En segundo lugar, revisar las diligencias para identificar los contactos y las reuniones que habían mantenido entre sí los miembros de las organizaciones durante la

\footnotetext{
${ }^{9}$ Los datos que ser recogieron sobre el perfil de los miembros del grupo a partir de la información disponible son los siguientes: sexo, edad, estado civil, situación laboral, nacionalidad, lugar de residencia, posición jerárquica en la organización, función principal realizada en la organización y antecedentes policiales relacionados o no con la delincuencia organizada.
} 
investigación. Esta segunda fuente de información era necesaria para llevar a cabo el ARS y comprobar: la estructura de los grupos, los miembros principales y sus posiciones en el interior de las organizaciones. La fuente de datos utilizada (información policial), siendo una fuente valiosa de información y muy difícil de obtener, también adolece de grandes limitaciones citadas reiteradamente en la literatura (Krebs, 2002 y Sparrow, 2001): a) los datos procedentes de la investigación criminal son incompletos y sesgados por la investigación; b) las barreras entre lo legal y lo ilegal son difíciles de distinguir, complicando el proceso de selección de los miembros activos de la red; y c) las redes encubiertas suelen ser dinámicas y ello no suele reflejarse en los datos recogidos, que reflejan datos y realidades estáticas. El principal escollo de la investigación que aquí se presenta es que la información disponible de las fuentes policiales sobre los contactos es incompleta porque únicamente se dispone de los contactos identificados en la investigación policial, esto impide que los resultados obtenidos de su análisis sean concluyentes y científicamente contrastados. Sin embargo, nuestra pretensión principal no es obtener resultados concluyentes acerca de cada una de las redes, sino comparar la información obtenida de la investigación policial y la aportación que el ARS puede proporcionar sobre los mismos datos.

El registro de contactos se realizó en una plantilla donde se recogía la fecha del contacto telefónico, el emisor y el receptor de la comunicación, así como la duración de la misma. Dicha información luego fue trasladada a matrices de modo 1 que fueron a analizadas a través del software UCINET 6 y Netdraw para realizar los grafos.

\subsection{Análisis de la estructura de las redes}

El análisis de la estructura de un grupo de crimen organizado es una tarea compleja dada la variedad de medidas cualitativas y cuantitativas que utilizan los investigadores para dicho propósito (Natarajan, 2000). Sin embargo, el ARS permite algunos análisis que, si bien no nos permiten analizar la estructura, aportan algunos índices útiles a la hora de valorar la densidad y centralidad de las organizaciones, constituyendo formas alternativas al estudio tradicional basado en las posiciones jerárquicas de sus componentes. En su lugar, el análisis de redes sociales otorga pesos específicos a las relaciones existentes entre los miembros de una red, permitiendo identificar los 
miembros más relevantes según el nivel de contactos que mantienen con los demás componentes del grupo. Finalmente, también permite la identificación de subgrupos o grupos dentro de la red.

El primer objetivo de la investigación consistía en analizar la estructura de las cuatro organizaciones según el ARS, para comprobar si la información proporcionada por la policía, de acuerdo con los archivos de investigación de las diferentes operaciones, era coincidente. Con este objetivo, se ha comparado la información sobre la estructura de los grupos proporcionada por los archivos policiales (interpretación policial) y la información proporcionada por el ARS (a partir de la matriz de contactos de las cuatro redes analizadas). Los análisis que se han realizado para dicho objetivo son los siguientes: centralización de la red, densidad del grupo y el centro/periferia del grupo (Borgatti y Everett, 1999).

El segundo objetivo de la investigación consistía en analizar los actores principales de la red. Este análisis ha permitido examinar si las conclusiones aportadas por el ARS eran coincidentes con las interpretaciones policiales aportadas por los archivos de la investigación policial. Para ello se han llevado a cabo análisis de centralidad e intermediación para conocer los actores con mayor poder e intermediación de la red. El grado de centralidad mide el número de contactos directos entre un actor y el resto de actores de la red. El grado de intermediación de un sujeto mide el peso de la intermediación de los actores en una red ${ }^{10}$ (Sparrow, 1991). Asimismo, se han calculado otras medidas de centralidad complementarias como la cercanía (Closeness) y el eigenvector de distancias geodésicas. La cercanía mide los actores que están conectados a muchos actores bien conectados y el eigenvector identifica los actores más centrales en términos de la estructura global de la red.

El tercer objetivo de la investigación pretendía conocer las posiciones jerárquicas de la mayor parte de los miembros con el fin de contrastar la percepción policial respecto a la estructura jerárquica de la red. Este análisis se ha hecho calculando el grado de centralidad de cada uno de los miembros clasificados según su posición jerárquica. Así, podíamos ver si la clasificación proporcionada por la policía

\footnotetext{
${ }^{10} \mathrm{El}$ grado de centralidad, se basa en el número de contactos directos entre un actor y el resto de actores de la red. El grado de intermediación indica la frecuencia con la que un nodo aparece en el camino más corto que conecta otros dos nodos (Sparrow, 1991).
} 
correspondía con la posiciones ofrecidas por el ARS según el peso de los sujetos en las relaciones internas de la red.

A continuación presentaremos los resultados que hacen referencia a los objetivos marcados en la investigación.

\section{Resultados}

\subsection{Operación $M$}

\section{Estructura de la red}

De acuerdo con la información proporcionada por los archivos de la policía, el grupo principal investigado (importador) en la Operación M estaba relacionado con dos grupos: un grupo de nivel medio español encargado de transportar, almacenar y adulterar la droga, y un grupo de nivel inferior o minorista a cargo de la distribución de la cocaína desde Madrid a otros puntos como Valencia. La investigación policial concluyó que el grupo investigado (en su totalidad ${ }^{11}$ ) tenía una estructura jerarquizada y compleja, siendo éste un indicador de su peligrosidad ${ }^{12}$.

Cuando se llevó a cabo el ARS sobre el grupo en su conjunto, los resultados encontrados no fueron del todo similares. De acuerdo con las medidas proporcionadas por el ARS, la red tenía una centralización del 5,21\% y una densidad del 13\%, con una desviación típica de 1,35. En este caso, la densidad encontrada es baja en comparación con otras redes similares (Network-Ciel- Morselli, 2009). Por último, si se calcula el núcleo y la periferia de la red, el resultado nos proporciona que lo forman 7 miembros (PCCP, JPPM, HPM, SNRM, JDD, HAMS y WGV) de un total de 31 sujetos investigados, lo que significa que el $32 \%$ de los miembros tienen una fuerte relación dentro de la estructura en general. En definitiva, la descripción encontrada, teniendo en cuenta que los datos de los contactos son incompletos y que el análisis se realizó sobre el conjunto de los tres grupos, no confirma del todo la estructura compleja y jerarquizada apreciada. De ser así debería tener un alto nivel de centralidad y, en

${ }^{11} \mathrm{El}$ análisis de redes sociales se ha realizado con los grupos detectados en la operación policial. Al comparar la información de la policía con los análisis de redes sociales, hemos considerado que el análisis global era la mejor opción para proceder a dicha comparación. Sin embargo, somos conscientes de que este hecho puede afectar a los resultados obtenidos.

${ }^{12}$ De cada operación policial se recogió el nivel de riesgo de la misma percibido por el investigador así como los indicadores que según él permitían otorgarle dicho nivel de riesgo. 
cambio, la red tiene una densidad baja y su núcleo está compuesto por un amplio grupo de miembros que comparte un patrón de relación similar.

La figura número1 muestra la representación de la red M proporcionada por el ARS, que incluye tres organizaciones mostradas en distintos colores. En azul se marcan los clientes de esta organización.

\section{Figura 1. Representación gráfica de la Red M $(N=22)$}

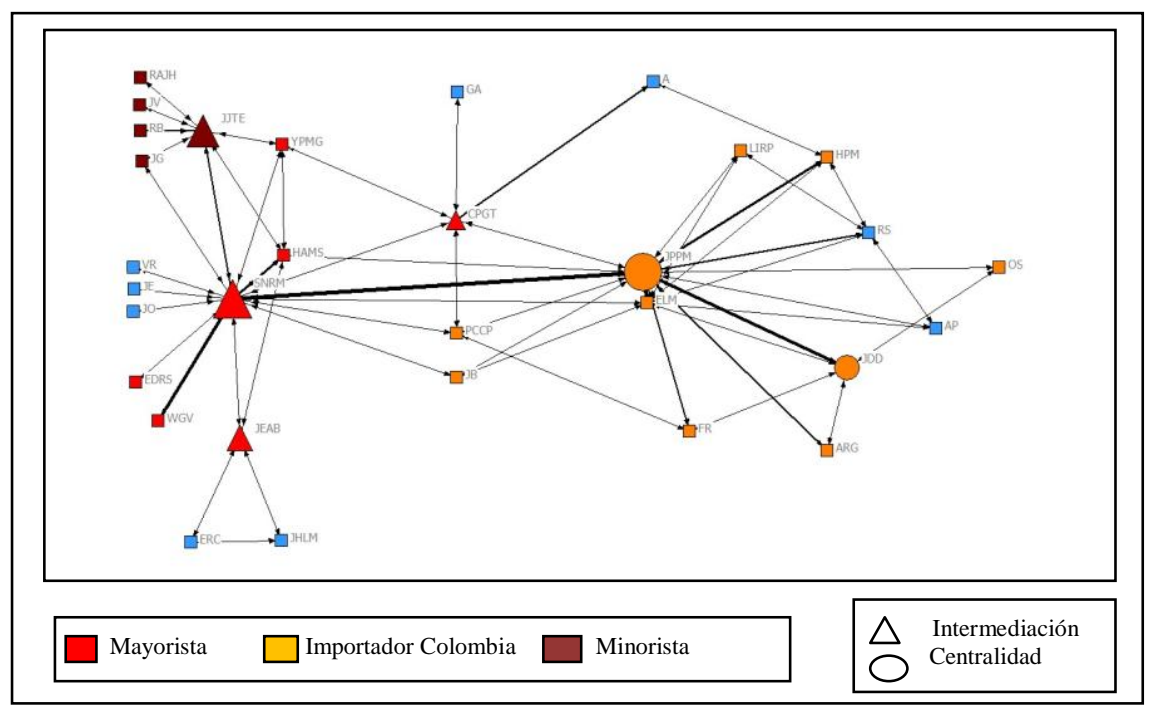

Los actores principales y su posición dentro de la organización.

La figura número 1 también muestra los diferentes miembros que desempeñan un papel clave (centralidad e intermediación) dentro de la red (ver figura 1 y tabla 2). En primer lugar, la persona con mayor grado de centralidad e intermediación es SNRM. Se trata del líder del grupo mayorista local cuyo papel bisagra de distribuidor a diferentes grupos locales le otorga también el rol del intermediario, corroborado por su alto grado de intermediación (tabla 2). Esto es, su papel de intermediario en España deriva de su posición en el mercado como proveedor de cocaína de varios minoristas en el mercado local español. SNRM también presenta un mayor grado cercanía al resto de la red, lo que sugiere que existe una mayor facilidad de acceso al resto de la red para obtener y enviar información.

En segundo lugar, en términos de centralidad e intermediación tenemos a JPPM (líder del grupo importador), muy cercano a SNRM en grado de centralidad. JPPM es 
un varón de 60 años, ecuatoriano, con residencia permanente en Madrid. Si vemos la distancia geodésica comprobamos que es él y no SNRM quien tiene en términos globales una posición más central y una mejor conexión a nodos bien conectados. Por tanto, podemos identificar a JPPM como un actor central dentro de la red y de mayor exposición y riesgo dentro de la misma.

Además de los dos miembros anteriores, CPGT (figura n. 1) es también un miembro relevante como coordinadora de JPPM. Ella tiene un bajo nivel de intermediación, pero juega un papel clave entre los dos grupos de la red. A continuación vemos reflejadas en la tabla número 2 las principales medidas de centralidad de los miembros de la red.

Tabla 2. Medidas de centralidad de los miembros más importantes de la red.

\begin{tabular}{|l|l|l|l|l|}
\hline Miembros & $\begin{array}{l}\text { In degree } \\
\text { Centralidad } \\
\text { normalizada }\end{array}$ & $\begin{array}{l}\text { NBetweenness } \\
\text { Intermediación } \\
\text { normalizada }\end{array}$ & $\begin{array}{l}\text { Ncloseness } \\
\text { Cercanía } \\
\text { normalizada }\end{array}$ & Neigenvector \\
\hline SNRM & $\mathbf{5 . 5 3 7}$ & $\mathbf{1 2 . 4 4 0}$ & $\mathbf{3 . 0 1 3}$ & $\mathbf{8 2 . 9 8 2}$ \\
\hline JPPM & $\mathbf{5 . 4 8 2}$ & $\mathbf{6 . 5 2 9}$ & $\mathbf{3 . 0 0 2}$ & $\mathbf{8 6 . 7 1 3}$ \\
\hline $\boldsymbol{H P M}$ & 1.425 & 0.496 & $\mathbf{2 . 9 7 2}$ & 31.138 \\
\hline JJTE & 1.700 & $\mathbf{4 . 3 5 5}$ & 2.987 & 17.612 \\
\hline JDD & 1.371 & 0.094 & 2.969 & $\mathbf{3 7 . 8 7 3}$ \\
\hline JEAB & 0.164 & 0 & 2.975 & 4.861 \\
\hline $\boldsymbol{C P G T}$ & 0.713 & 2.781 & 2.995 & 4.038 \\
\hline
\end{tabular}

Además de los miembros clave en la red, el estudio de la investigación policial mostró una distribución convencional jerárquica entre los miembros de la red. Los miembros más relevantes son los líderes de los tres grupos organizados (importadorJPPM, mayorista- SNRM y minorista-JJTE), por debajo estan los coordinadores (FT, OS, FR, HPM, ELM y PCCP) y en el nivel más bajo de los grupos se encuentran las tareas operativas tales como el transporte, la adulteración, distribución y protección llevadas a cabo por los siguientes miembros: JDD, VAD, ELF, WGV, HAMS y YPMG.

A partir de los análisis de las posiciones jerárquicas realizados en el marco del ARS, (ver figura n. 2) llegamos a la conclusión de que los resultados confirman la 
concepción jerárquica procedente de la información policial. Esto es, los miembros con un alto grado de centralidad son los que tienen posiciones jerárquicas superiores en la red. En algunos casos, ciertos miembros (JDD y YPMG) tienen un mayor grado de centralidad respecto a la posición jerárquica descrita en la investigación, aunque la diferencia es insignificante. Sin embargo, es importante destacar nuevamente el caso de CPGT. CPGT es la esposa de SNRM y en los archivos de la policía se considera un miembro que tiene una posición secundaria o auxiliar. Sin embargo, el ARS muestra que tiene un papel mucho más clave dentro de esta red de acuerdo a su grado de centralidad. Esta situación se aprecia perfectamente en la figura número 2.

Figura 2. Distribución de las posiciones jerárquicas y grado de centralidad en la red $M^{13}$

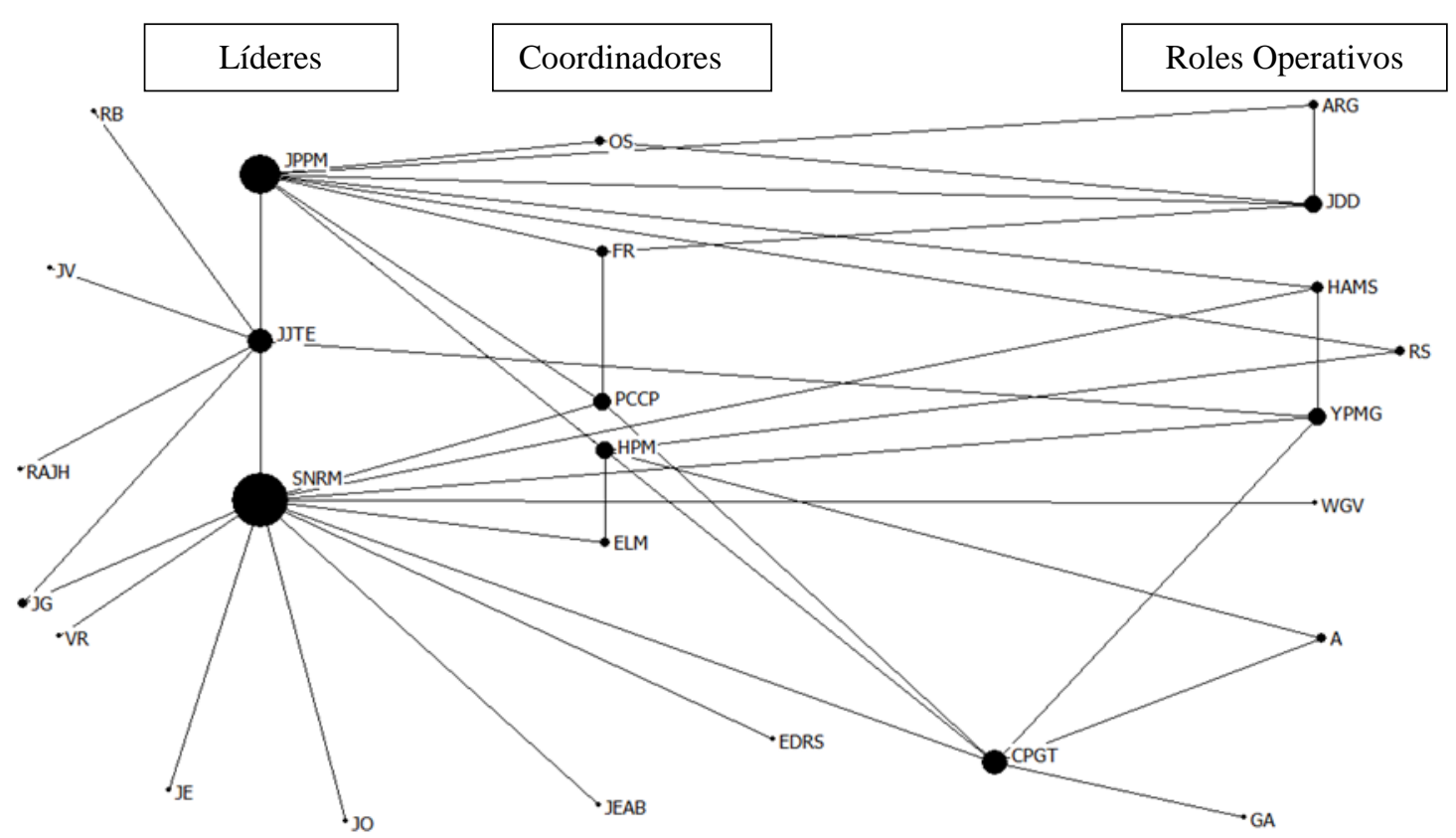

\subsection{Operación J}

\section{Estructura de la red}

En la red $\mathbf{J}$ se observan tres organizaciones delictivas autónomas de acuerdo con los archivos policiales. La operación policial llevada a cabo sobre estos grupos se inició con la investigación del grupo encargado del blanqueo de dinero que, posteriormente, condujo a la identificación de otros dos grupos: un grupo importador

${ }^{13}$ El tamaño de los círculos representa el mayor o menor grado de centralidad de los sujetos. 
liderado por JLT, que traía la cocaína de Méjico a España y, otro grupo mayorista, liderado por OJSR, que se encargaba de la distribución de cocaína en la península. Desde los archivos policiales quedaba claro que la organización principal era el grupo encargado del transporte ilegal de cocaína de México (importador) para ser distribuido por otro proveedor al por mayor en España. Este grupo fue clasificado por la policía como altamente peligroso por la posesión de armas de algunos de los miembros y por su peligrosidad. Por el contrario, el grupo cuya principal tarea era blanquear el capital ilegal de otros grupos, llevaba a cabo dichas tareas a través de una joyería que se utilizaba como empresa pantalla para ocultar las actividades ilegales. El líder (EHJ) contaba con algunos colaboradores cercanos y con gran cantidad de sujetos que cambiaban billetes de bajo valor por billetes de alto valor con el fin de enviarlo físicamente a los países de origen.

Para comprobar las características de la red, se procedió a realizar el ARS de toda la red, incluyendo el grupo dedicado al blanqueo de capitales ${ }^{14}$. Los resultados obtenidos condujeron a una centralidad muy baja (6,8\%) y una densidad muy baja (10\%), con una desviación típica de 0,81 . Por otra parte, el núcleo del grupo está compuesto por 19 miembros, de un total de $49(39 \%)$. Ello significa que aproximadamente el $40 \%$ de la red posee contactos continuos, existiendo un flujo de información permanente entre ellos. Estos resultados no confirman del todo una percepción jerárquica respecto a la estructura de la red (representada en la figura 3) que distingue dos grupos autónomos con diferentes jerarquías. A través de este análisis se observa cómo las relaciones entre los dos grupos son más intensas en la realidad y que los vínculos entre las dos organizaciones dedicadas al tráfico de cocaína, no se canalizan a través de sus principales dirigentes (OJSR y JLT): las conexiones se producen directamente entre los sujetos de diferentes grupos.

\footnotetext{
${ }^{14}$ El hecho de realizar los ARS sobre varios grupos puede afectar a los resultados y sobredimensionar la descentralización de la red. Sin embargo, esta decisión inicial obedece a dos motivos: en primer lugar, porque la estructura aportada por la policía suele referirse al conjunto de los grupos que se identifican en la operación. En segundo lugar, porque los contactos telefónicos no se obtienen de todos los sujetos identificados en la operación policial y las medidas de centralidad e intermediación están limitadas cuando el análisis se realiza sobre una muestra de pequeño tamaño.
} 
Figura 3. Representación gráfica de la red $J(N=49)$

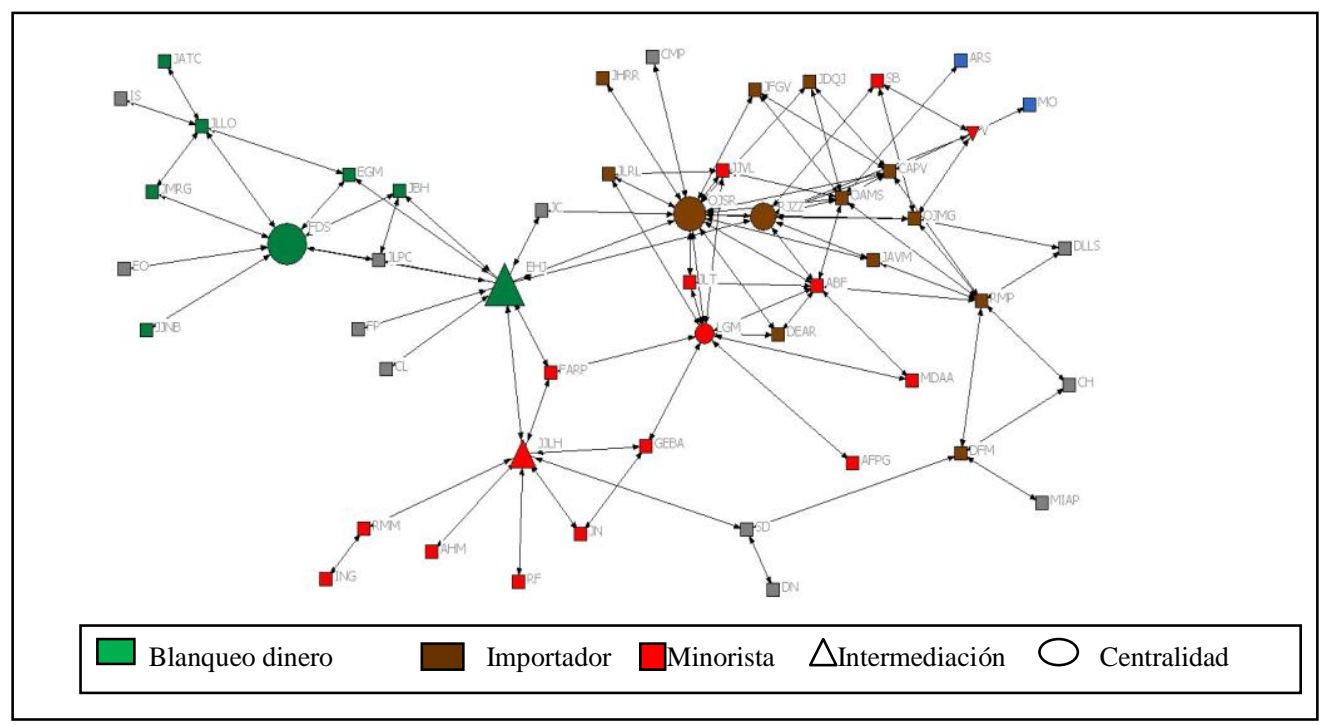

\section{Los principales actores y posiciones jerárquicas dentro de la red $\mathrm{J}$}

La identificación de los actores clave de la red según los análisis de centralidad e intermediación mostrados en la tabla número 3, nos permite llegar a los siguientes resultados: los miembros más relevantes de la red forman parte de la organización de blanqueo de dinero, a pesar de que el tráfico de drogas sea la principal actividad percibida. Como muestra la tabla 3, FDS es el actor más central de la red, con el grado de centralidad más alto y un mayor grado de eigenvector. En este caso, FDS es un hombre de 31 años de edad, coordinador y mano derecha de EHJ, que se encarga del transporte y la distribución de las tareas necesarias para el blanqueo. En términos de intermediación, EHJ se encuentra en primer lugar. Es el líder del grupo y el propietario de la joyería que ofrece la tapadera para el blanqueo del dinero obtenido del tráfico de cocaína. Finalmente, OJSR (líder de la organización mayorista de cocaína en España) también es un actor clave dentro de la red ya que ocupa el segundo lugar en términos de centralidad e intermediación. La siguiente tabla muestra los resultados más significativos. 
Tabla 3. Medidas de centralidad de los miembros más importantes de la red $(N=49)$

\begin{tabular}{|l|l|l|l|l|}
\hline Miembros & $\begin{array}{l}\text { NrmIndegree } \\
\text { Centralidad } \\
\text { normalizada }\end{array}$ & $\begin{array}{l}\text { NrmBetweennes } \\
\text { Intermediación } \\
\text { normalizada }\end{array}$ & $\begin{array}{l}\text { NCloseness } \\
\text { Cercanía } \\
\text { normalizada }\end{array}$ & NEigenvector \\
\hline $\boldsymbol{F D S}$ & $\mathbf{5 . 7 8 1}$ & 9.175 & 4.928 & $\mathbf{9 7 . 6 5 5}$ \\
\hline $\boldsymbol{O J S R}$ & $\mathbf{4 . 2 6 0}$ & $\mathbf{1 8 . 2 7 6}$ & $\mathbf{5 . 0 5 2}$ & 13.547 \\
\hline $\boldsymbol{R J Z Z}$ & 2.130 & 7.334 & 5.015 & 3.212 \\
\hline $\boldsymbol{L G M}$ & 2.028 & 6.143 & 4.967 & 1.364 \\
\hline $\boldsymbol{E H J}$ & 2.333 & $\mathbf{2 6 . 5 4 4}$ & $\mathbf{5 . 0 5 2}$ & $\mathbf{4 6 . 2 1 3}$ \\
\hline $\boldsymbol{J J L H}$ & 1.318 & 14.117 & 4.971 & 1.194 \\
\hline
\end{tabular}

Una vez identificados y definidos los actores con mayor poder de la red, analizaremos la relación entre la posición de centralidad de los miembros y las posiciones jerárquicas otorgadas a cada miembro desde la investigación policial. Ya hemos comentado que los archivos policiales identifican dos estructuras jerárquicas independientes, una dedicada al tráfico de cocaína y otra subcontratada para actividades de blanqueo de capitales. La red dedicada al tráfico de cocaína estaba compuesta por tres grupos: un grupo importador dirigido por JLT, quien contaba con coordinadores fieles como FARP, JJVL, AFPG y LGM. JLT, a su vez, también estaba en contacto con el grupo dedicado a la distribución de droga dirigido por OJSR y coordinado por JFVG, CAPV, RJZZ y OJMG. El tercer grupo estaba a cargo del transporte, la protección y la logística a través de SD, JHRR, DFM, RMP y JAVM. Finalmente, la red dedicada al blanqueo de dinero mostraba, según la percepción policial, una estructura más horizontal integrada por un líder (EHJ), un coordinador (FDS), tres miembros $\begin{array}{lllll}\text { encargados de recolectar el dinero (JBH, JLLQ y EGM) y } & 12\end{array}$ miembros colaboradores a cargo del blanqueo de capitales a través del territorio español.

La comparación entre posiciones jerárquicas ocupadas por los miembros y el grado de centralidad sobre la red $\mathbf{J}$ no ofrece una distinción clara entre los dos grupos ya que ambos mantienen numerosas conexiones a muchos niveles. Tampoco el grado de centralidad de algunos miembros se corresponde con la posición y el papel que 
parece desprenderse de la investigación. Algunos líderes, como JLT, parecen tener un menor grado de centralidad según los resultados del ARS y muchos miembros de nivel medio o bajo tienen un grado inferior o superior de centralidad según las relaciones que poseen en la organización: ABF, RMP, FARP, JJVL y EGM. La siguiente figura muestra esas diferencias.

Figura 4. Distribución de roles según el grado de centralidad en la red J

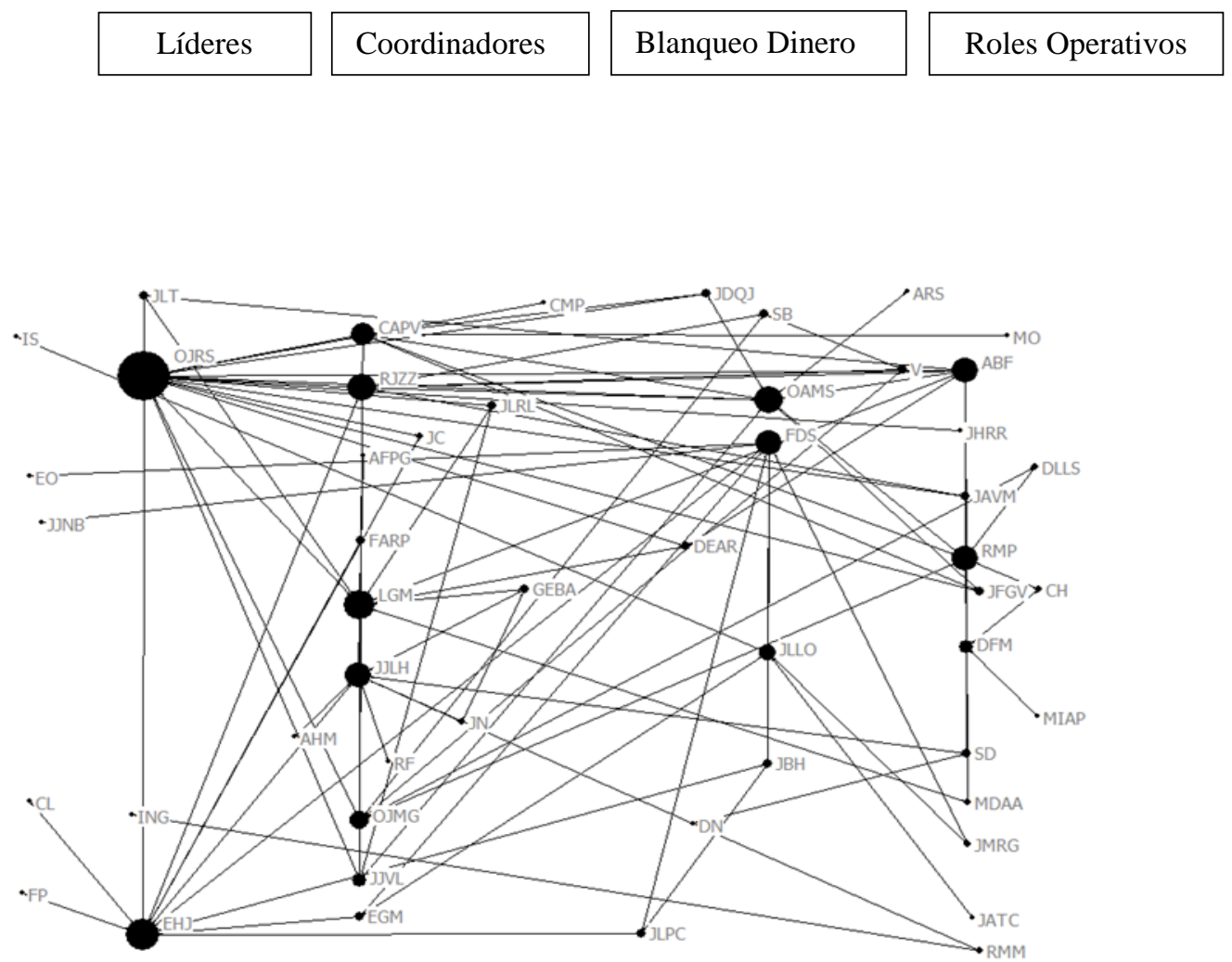

\subsection{Operación $K$}

Estructura de la red

Esta operación permitió la desarticulación de un grupo distribuidor de cocaína y heroína (mayorista y minorista) que desarrollaba sus actividades mercantiles en una amplia zona de Madrid. Este grupo tenía, a su vez, relación con otros tres grupos: a) dos grupos que distribuían cocaína localmente, al por mayor o al por menor y b) un grupo que, por lo que sabemos, sólo se dedicaba a la venta al por mayor de heroína. El grupo principal está fuertemente unido por vínculos familiares y étnicos (gitanos 
españoles) y pertenece a un colectivo que ha estado tradicionalmente relacionado con la distribución de heroína en áreas marginales de las grandes ciudades. JFM es el líder del clan y el responsable de la distribución de la cocaína importada desde Perú por el grupo liderado por RBM. De acuerdo con la investigación policial, este grupo familiar poseía características típicas de un grupo mayorista español de cocaína y heroína: una estructura jerarquizada y fuertes lazos familiares entre sus miembros. En el momento de la investigación, su líder (JFM) se encontraba en la cárcel por delitos previos cometidos en el 2008. Sin embargo, este hecho no le impidió coordinar sus negocios a través de dos de sus hermanos (principalmente a través de JES) y de su hijo ABFM. En la red jerárquica presentada por la policía, podemos ver una organización con un pequeño núcleo central formado por los miembros que mantienen lazos de sangre (JFM, JES, AFM y ABFM). Estos se encuentran apoyados por otros colaboradores con papeles secundarios.

Por el contrario, de acuerdo con el ARS realizado, la red revela una centralización de $3.3 \%$ y una densidad del $8 \%$, con una desviación típica de 0.86 . El núcleo está compuesto por 18 miembros de un total de 41 (55\%). Estos resultados muestran una estructura horizontal mucho más flexible que la descrita con anterioridad con media red que mantiene intensas relaciones entre sí

Figura 5. Representación gráfica de la Red $K(N=33)$

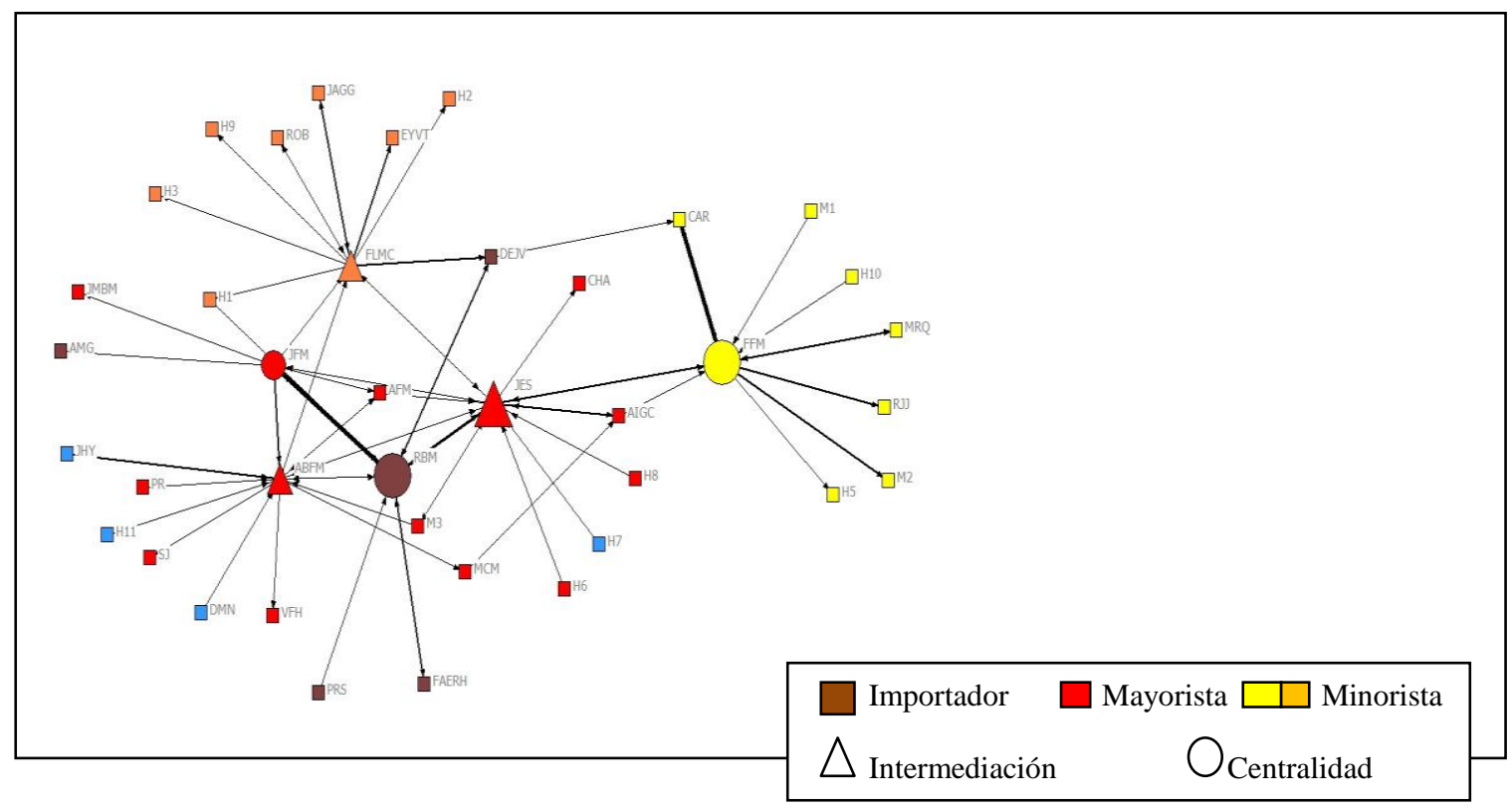


Los principales actores y sus posiciones dentro de la organización

Según se observa en la figura número 5 y la tabla numero 4, FFM y RBM son los miembros de la red con mayor grado de centralidad (con valores muy semejantes), aunque RBM (líder grupo importador) figura en primer lugar cuando calculamos la centralidad en términos globales (eigenvector). El primero de ellos, FFM (líder grupo distribuidor), es un varón español de 49 años, de etnia gitana que, además de ser el principal cliente de cocaína de JES (tercer puesto en centralidad), se encarga también de distribuirla en su barrio residencial y otros puntos de España. El segundo miembro relevante es RBM, principal importador de cocaína de la organización dirigida por JFM. RBM es un varón de origen dominicano, de 38 años de edad, de nacionalidad española y con residencia permanente en Madrid. El cuarto puesto de centralidad se consagra al líder de la organización mayorista (JFM) quien, curiosamente, se encuentra en prisión cumpliendo una pena por una causa anterior. Este hecho es especialmente relevante y muestra cómo el encarcelamiento no constituye un obstáculo para el mantenimiento de contactos fuera de la cárcel ni un impedimento para la dirección y coordinación del negocio ilegal. Los datos sobre las medidas de centralidad de los principales sujetos se muestran en el cuadro número 4. $(N=41)$

Tabla 4. Medidas de centralidad de los miembros más importantes de la red

\begin{tabular}{|l|l|l|l|l|}
\hline Miembros & $\begin{array}{l}\text { NrmIndegree } \\
\text { Centralidad } \\
\text { normalizada }\end{array}$ & $\begin{array}{l}\text { NBetweenness } \\
\text { Intermediación } \\
\text { normalizada }\end{array}$ & $\begin{array}{l}\text { NCloseness } \\
\text { Cercanía } \\
\text { normalizada }\end{array}$ & NEigenvector \\
\hline FFM & $\mathbf{3 . 6 5 1}$ & 12.541 & 4.201 & 58.182 \\
\hline $\boldsymbol{R B M}$ & $\mathbf{3 . 4 2 8}$ & 6.530 & 4.187 & $\mathbf{7 4 . 0 1 2}$ \\
\hline $\boldsymbol{J E S}$ & $\mathbf{2 . 9 0 6}$ & $\mathbf{2 2 . 1 8 7}$ & $\mathbf{4 . 2 6 6}$ & 52.031 \\
\hline $\boldsymbol{J F M}$ & $\mathbf{2 . 3 8 5}$ & 5.796 & 4.230 & $\mathbf{6 2 . 6 4 2}$ \\
\hline $\boldsymbol{F L M C}$ & 1.788 & $\mathbf{1 4 . 3 5 9}$ & $\mathbf{4 . 2 3 3}$ & 16.894 \\
\hline $\boldsymbol{C A R}$ & 1.714 & 0.191 & 4.099 & 46.897 \\
\hline $\boldsymbol{A B F M}$ & 1.714 & 13.189 & $\mathbf{4 . 2 3 6}$ & 19.191 \\
\hline $\boldsymbol{D E J V}$ & 0.820 & 0.583 & 4.147 & 21.397 \\
\hline
\end{tabular}


La tabla 4 también nos muestra los miembros que resultan relevantes según su grado de intermediación o su posición de brokers dentro la red. El mayor grado de intermediación es ocupado por JES, actual jefe de la organización mayorista. JES es un varón de 42 años de edad, español y con un amplio historial de antecedentes policiales por tráfico de drogas, robo y violencia. Él es también el encargado de ponerse en contacto con los proveedores y de distribuir la droga, mientras que su hermano (JFM) está en la cárcel. Po otra parte, en esta red, cuyos vínculos familiares priman sobre otros motivos para otorgar las funciones y posiciones relevantes en la organización, debemos resaltar la existencia de otro miembro familiar: el hijo de JFM. Éste aparece con un grado relevante de intermediación $\left(3^{\circ}\right)$, contando con tan solo 14 años de edad. Ello se debe a que realizaba funciones similares a las de su tío JES.

La relación entre posiciones jerárquicas dentro de la red y el grado de centralidad de los distintos miembros de la red no ofrece demasiadas sorpresas en relación con la investigación criminal. La siguiente figura (número 6) muestra la distribución de roles en función del grado de centralidad de los miembros más relevantes. La tendencia general no difiere de la descripción proporcionada por la policía ya que las personas con mayor grado de centralidad ocupan posiciones jerárquicas acordes con su importancia. En este caso, los miembros con mayores vínculos familiares tienen medidas similares y muy homogéneas debido a su posición y función intercambiable.

También podemos ver claramente en la figura siguiente una cuestión ya comentada: el papel relevante de JFM que, incluso desde la cárcel, seguía manteniendo sus contactos y garantizaba la inmunidad para su organización mediante el soborno de dos agentes de policía. En este caso, vemos un claro ejemplo del modo en que una red ilegal se ajusta cuando el líder es detenido y eliminado de la red. Los roles intercambiables de los tres miembros familiares permiten a la red adaptarse y reorganizarse después de la detención de su máximo jefe (JFM, JES y AFM). 
Figura 6. Distribución de roles por grado de centralidad en la red $K$

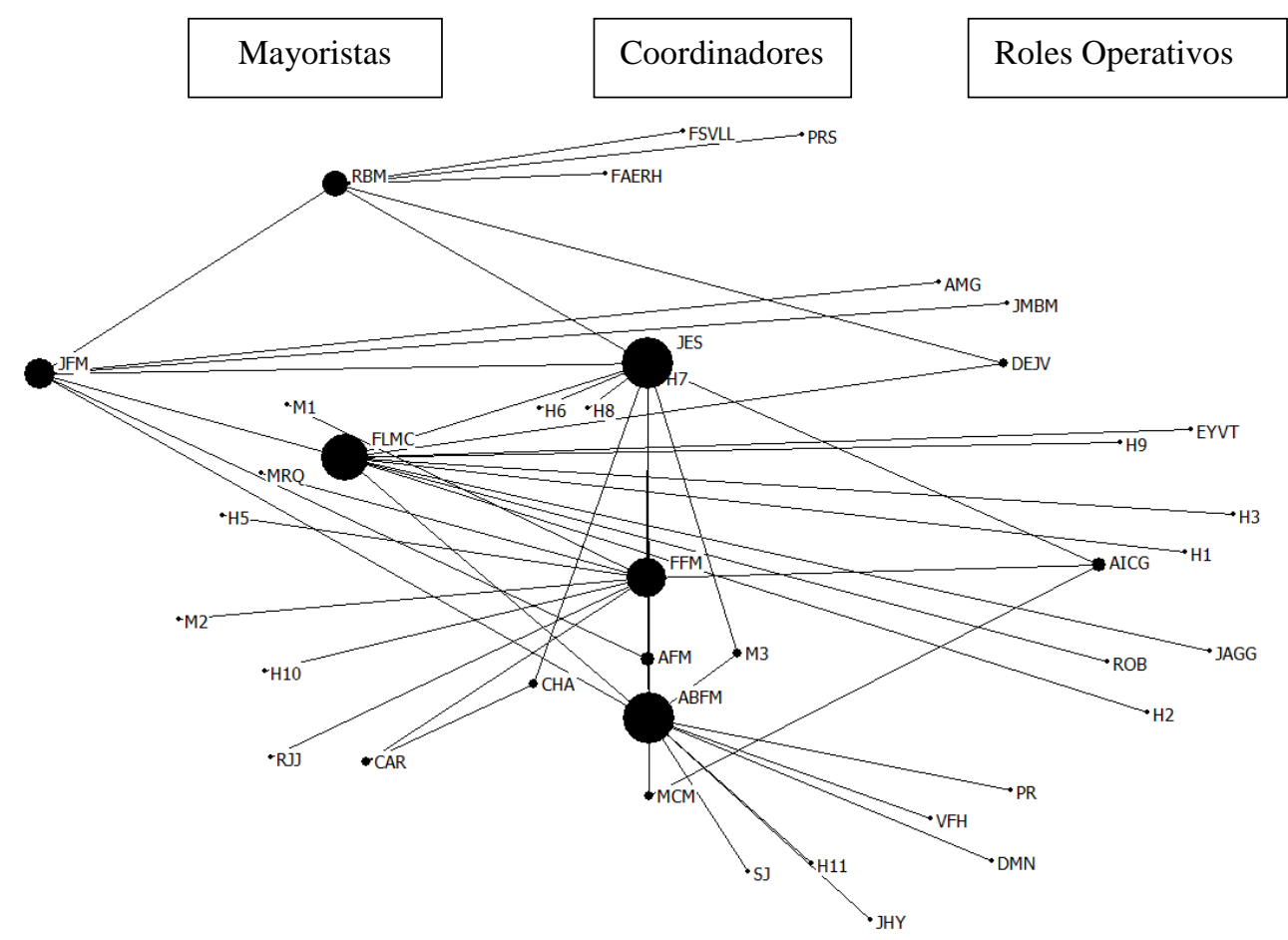

\subsection{Operación A}

\section{Estructura de la red}

La red descrita en la figura número 7 hace referencia a una familia española que configura un grupo de mayoristas dedicados a la venta de cocaína, procedente de Colombia, en tres provincias españolas. JFS es el principal importador de Colombia que vende la cocaína a SAR, quien asume la dirección de la organización en España. SAR cuenta con un colaborador y hombre de confianza (JEPO) que le ayuda en el transporte de la droga y la recolección del dinero procedente de la venta de la misma. Según la investigación policial, el grupo era un pequeño grupo familiar con una estructura compleja y un alto perfil de riesgo. Este riesgo se encontraba acreditado por el uso de armas, la compleja estructura y la peligrosidad de sus miembros.

La información procedente de la investigación policial también muestra una estructura jerárquica centralizada en un único líder (SAR). Esta jerarquía también se manifiesta en el mismo sentido al realizar el ARS sobre los contactos de los miembros 
de la red. Los análisis de centralización y densidad muestran una red centralizada y jerárquica, en comparación con las otras tres redes analizadas. La red de la Operación A tiene el mayor índice de centralización $(15,5 \%)$ y una alta densidad (54\%) con una Desviación Típica de 2,06. El núcleo está compuesto de tres miembros (MMM, SAR y JEPO), de un total de 11 miembros (27\%). Así, los resultados muestran una red con perfil de estructura jerárquica y tradicional, con SAR como único líder.

Figura 7. Representación gráfica de la Red A $(N=11)$

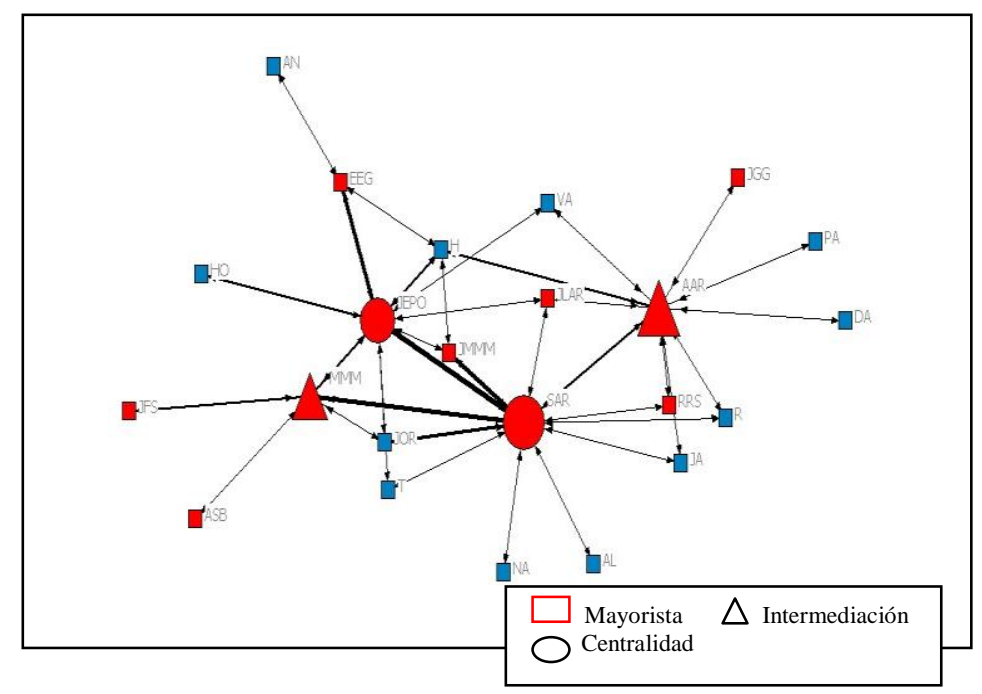

Los principales actores y sus posiciones dentro de la organización.

Este pequeño grupo familiar tiene una composición distinta al resto de las redes. Al tener SAR un papel tan central, asume tanto el rol de centralidad como el de intermediación. Como podemos observar en la tabla número 5, SAR tiene el mayor grado de centralidad, cercanía, eigenvector y de intermediación. SAR, de 44 años de edad, se encuentra a cargo del grupo y mantiene contactos personales con los importadores e cocaína. Tiene un papel autónomo en relación con el resto del grupo, a excepción de su fiel colaborador JEPO. En referencia a la intermediación, debemos comentar que AAR (hermana de SAR), está a cargo de la distribución de cocaína junto con su pareja, ocupando el segundo lugar. La tercera mujer involucrada en la red es la madre de SAR (RRS), quien se encuentra a cargo de la gestión y el cobro del dinero procedente del negocio ilegal. 
Tabla 5. Medidas de centralidad de los miembros más importantes de la red $(N=23)$.

\begin{tabular}{|l|l|l|l|l|}
\hline Miembros & $\begin{array}{l}\text { NormIndegree } \\
\text { Centralidad } \\
\text { normalizada }\end{array}$ & $\begin{array}{l}\text { NBetweenness } \\
\text { Intermediación } \\
\text { normalizada }\end{array}$ & $\begin{array}{l}\text { NCloseness } \\
\text { Cercanía } \\
\text { normalizada }\end{array}$ & NEigenvector \\
\hline SAR & $\mathbf{1 7 . 9 2 9}$ & $\mathbf{4 1 . 5 5 8}$ & $\mathbf{6 6 . 6 6 7}$ & $\mathbf{8 9 . 6 6 4}$ \\
\hline AAR & 6.061 & $\mathbf{3 2 . 7 9 2}$ & 57.895 & 23.547 \\
\hline JEPO & $\mathbf{1 1 . 6 1 6}$ & 26.082 & $\mathbf{5 9 . 4 5 9}$ & $\mathbf{6 9 . 9 8 4}$ \\
\hline $\boldsymbol{M M M}$ & 7.828 & 18.290 & 52.381 & 48.594 \\
\hline
\end{tabular}

En referencia al estudio de la relación entre centralidad y posición jerárquica, elaborado para las anteriores operaciones (figura número 8), los resultados mostrados por el ARS, nos ofrecen conclusiones similares a la estructura jerárquica derivada de la investigación. Como puede observarse en la figura número $8^{15}$, la distribución de posiciones según la centralidad encontrada coincide con el perfil de estructura descrito en la investigación policial. Sin embargo, esta tendencia se rompe con AAR ya que posee un alto grado de centralidad a pesar de ocupar posiciones menos centrales o inferiores dentro de la organización. Esta situación puede tener, al menos, dos explicaciones. En primer lugar, los vínculos familiares de los miembros de la red podrían explicar la adscripción de un miembro de la familia con mayor grado de centralidad a roles más básicos dentro de la red. En segundo lugar, el hecho de que el papel de las mujeres dentro de los grupos criminales haya sido tradicionalmente menos cualificado que el de los hombres, podría explicar que mujeres con un rol de baja cualificación ocupen una posición tan importante en términos de centralidad.

\footnotetext{
${ }^{15}$ En este caso hemos querido resaltar los miembros femeninos en color rojo por la especificidad de esta red en referencia a la participación y relevancia del género femenino en su composición.
} 
Figura 8. Distribución de roles según el grado de centralidad en la red Z

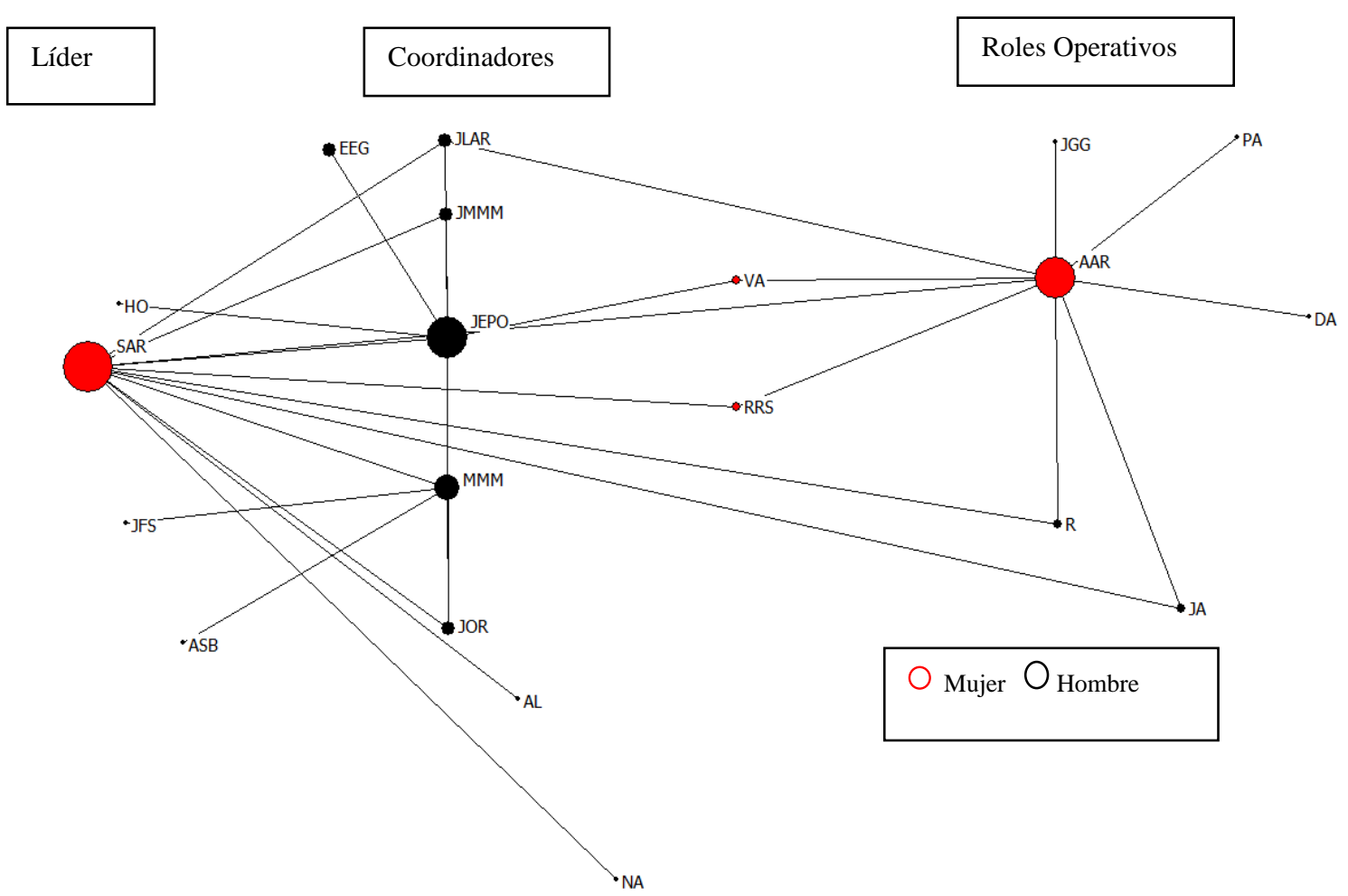

\section{Discusión}

El estudio de la estructura de los cuatro grupos criminales a partir de la comparación de los datos procedentes de la investigación policial y los resultados del ARS, nos lleva a la conclusión de que las estructuras descritas en las investigaciones policiales muestran mayores niveles de jerarquía y centralización. Esto es, los ARS realizados muestran estructuras más flexibles y horizontales, en comparación con los grupos clasificados por la policía como estructuras jerárquicas y complejas. Dichos resultados podrían explicarse por dos motivos: por un lado, porque el ARS se ha realizado sobre redes que incluyen varios grupos y ello podría explicar la mayor descentralización encontrada. Por otro lado, porque la fuente de datos utilizada se caracteriza por su limitación a los contactos detectados en la investigación, y ello impide el acceso a una visión global y completa de la red. En este sentido, la interpretación policial sobre la jerarquía y complejidad del grupo es el resultado de un 
juicio profesional más completo que se emite teniendo en cuenta múltiples variables además de los contactos entre sujetos. Por todo ello, consideramos que el ARS constituye una valiosa herramienta de apoyo en el contexto de la investigación criminal pero no puede, en ningún caso, sustituir al juicio del experto investigador.

Tras el análisis de las cuatro operaciones, tres de las redes (M, J y K) tienen porcentajes bajos de centralización y porcentajes muy bajos de densidad (por debajo del 10\%). Estos datos nos aproximan a perfiles de estructura reticulares con una división horizontal de funciones. Sin bien los resultados de este estudio no permiten, por la limitación de los datos, concluir acerca de la estructura de los grupos dedicados al tráfico de drogas, sí podemos decir que el perfil de estructura reticular es el más prevalente en la muestra de grupos mayoristas de cocaína analizados. También, tras el análisis pormenorizado de los grupos, hemos comprobado que éstos cumplen en mayor medida las características propias de las redes ilegales que de las organizaciones criminales (roles intercambiables, selección y adjudicación de función según lazos de sangre o étnicos, comunicación directa). La única excepción es la red A, que presenta una estructura más densa y centralizada, con un líder que comparte el mayor grado de centralidad e intermediación. Sin embargo, dicha jerarquía no se traduce posteriormente en una estructura de posiciones fijas, sino variables y dependientes de las acciones concretas requeridas. Por ello, podemos intuir que la estructura jerárquica y centralizada debería explicarse por el tamaño reducido de la red.

En referencia a los actores claves de las redes, el ARS ofrece la posibilidad de identificar a los miembros más relevantes de la red según su grado de centralidad, intermediación y cercanía. De los resultados obtenidos, dos de las redes poseen puntuaciones de centralidad e intermediación que no coinciden en la misma persona. Sin embargo, no podemos dar un patrón general respecto a los sujetos con mayor nivel de centralidad salvo que todos ellos lideran algún grupo. En las operaciones M y K, los miembros con las puntuaciones más altas en centralidad coinciden con los mayoristas e importadores de cocaína respectivamente. En la operación J, el miembro central es el segundo coordinador del grupo de blanqueo de capitales, mientras en la operación A es la líder del grupo de nivel medio o mayorista. 
Si hablamos de intermediación, los brokers de la red coinciden con los líderes de los grupos de nivel medio (mayoristas) encargados de recibir la mercancía y de su posterior venta a los minoristas. En dos de las operaciones, el intermediario es el líder del grupo mayorista en España. Por otro lado, en la operación J, el intermediario es el líder de la organización que se dedica a actividades de blanqueo de capitales para varias organizaciones. Finalmente, en la operación A y, debido a su pequeño tamaño, su líder es también el mayor intermediario. En este caso, sí se advierte un perfil mayoritario de broker coincidente con los líderes de los grupos mayoristas o intermediarios. Este resultado se encuentra en la misma dirección que otros estudios (Pearson and Hobbs, 2003; Desroches, 2005), que otorgan un papel bisagra esencial a los líderes de los grupos de nivel medio o mayoristas cuya desaparición o eliminación produce mayor daño y perjuicio a la red. Desde un enfoque estratégico, dichos sujetos deberían ser los sujetos prioritarios para la investigación policial.

Una vez realizado el análisis comparativo entre el grado de centralidad de los miembros de la red y las posiciones ocupadas por los mismos, los resultados del ARS confirmaron la percepción de las investigaciones policiales sobre las posiciones de los miembros. Sin embargo, en las figuras sobre las operaciones A y J, es interesante la observación de los actores que ocupan las posiciones jerárquicas más bajas en la organización. La posición de alguno de ellos no resulta acorde con su grado de centralidad según el ARS. En cambio, respecto a otros miembros que presentaban posiciones secundarias en la investigación, el ARS arroja una centralidad sensiblemente superior. Ello demuestra que este tipo de análisis aporta un valor añadido a las investigaciones policiales cuya finalidad es identificar los sujetos más relevantes de la red. El ARS permite identificar miembros que aparentemente se encuentran en puestos menos cualificados o en la periferia de la red pero son centrales si tenemos en cuenta los contactos que mantienen en el interior del grupo. Este tipo sujetos también constituyen los miembros más protegidos de la red por su difícil detección.

La integración de todos los resultados presentados en este artículo permite aventurarnos a establecer un perfil de cada una de las redes, según la clasificación mostrada en la parte inicial de este artículo. El primer grupo (M) es un clásico grupo de nivel medio o mayorista, cuyas personas claves en el negocio son el importador y 
el mayorista de cocaína en España. Este grupo podía ser calificado como una cooperativa, con una estructura horizontal y cuyos miembros comparten los recursos a su disposición para desarrollar, en común, la actividad ilegal (Haller, 1990). La operación K integra una empresa familiar cuya impronta familiar no sólo impregna el negocio ilegal sino que se refleja en su estructura y funcionamiento. Por otro lado, dicha actividad familiar se desarrolla en una comunidad que vive y convive con la actividad ilícita, lo que permite una mayor protección del grupo criminal. También el grupo liderado por la mujer (A) pude considerarse como una empresa familiar, con una estructura más jerarquizada cuyos vínculos se basan en la confianza y las relaciones familiares. Finalmente, el grupo dedicado al blanqueo de capitales puede ser un tipo más corporativo cuya actividad ilegal está profesionalizada y ofrecida a otros grupos.

Finalmente, los resultados presentados en este trabajo constituyen una pequeña prueba de las ventajas que ofrece el ARS para el análisis de los grupos criminales relacionados con el crimen organizado. Si bien las limitaciones propias de las fuentes de datos utilizadas (datos limitados, sesgados y parciales) impiden arrojar conclusiones sobre la estructura de los grupos dedicados a la venta de droga en nuestro país, podemos advertir que las organizaciones pertenecientes a la categoría de mayoristas tienen un perfil más propio de redes que de organizaciones tradicionales. Sin embargo, lo que sí podemos avanzar con este trabajo es que el ARS nos ofrece una herramienta de investigación útil para analizar este tipo de redes a partir de los elementos siguientes: personas claves dentro de la red criminal, la estructura y dinámica interna de la red, y la identificación de personas que ocupan posiciones relacionales más relevantes, independientemente de su función operativa o interna dentro del grupo.

\section{Referencias}

Adler, P. A. (1985). Wheeling and dealing: An ethnography of an upper-level dealing and smuggling community. New York: Columbia University Press.

Adler, P.A. (1992). The "post'" phase of deviant careers: Reintegrating drug traffickers. Deviant Behavior, 13, 103-126. 
Ayling, J. (2009). Criminal organizations and resilience. International Journal of Law, Crime and Justice, 37, 182-196.

Benson, J. S. y Decker, S. H. (2010). The organizational structure of international drug smuggling. Journal of Criminal Justice, 38, 130-138.

Borgatti, S.P. y Everett, M. G. (1999). Models of core/periphery structures. Social Networks, 21, 375-395.

Borgatti, S.P. (2006). Identifying sets of key players in a network. Computational, Mathematical and Organizational Theory. 12(1), 21-34.

Canter, D. (2004). A partial Order Scalogram Analysis of Criminal Network structures. Behaviormetrika, 31(2), 131-152.

Carley, K.M., Lee, J-S., Krackhardt, D. (2002). Destabilizing Networks. Connections, 24 (3), 31-34.

Coutu, D.L. (2002). How resilience works. Harvard Business Review 80(5), 4655.

Cressey, D. (1972). Criminal organizations: Its elementary forms. New York: Harper and Row.

DesRoches, F. (1999) Wholesale drug dealers, paper presented to panel on 'The structure and operation of illegal commodity markets'. Annual Meeting of the American Society of Criminology. Toronto.

Dorn, N., Murji, K., South, N. (1992). Traffickers: Drug markets and law enforcement. Routledge: London.

Fickenauer, J. O. y Waring, E.J. (1998). Russian Mafia in America: Immigration, Culture and Crime. Boston: Northeastern University Press.

Gambetta, D. (1988). Mafia: The Price of Distrust. En Gambetta, D. (ed). Trust making and breaking cooperative relations. Oxford: Blackwell, 158-175.

Hobbs, D. (2001). The Firm: Organizational Logic and Criminal Culture on a Shifting Terrain, British Journal of Criminology, 41, 549-560.

Horne, J.F. (1997). The coming age of organizational resilience. Business Forum 22(2/3), 24-28.

Ianni, F. (1972). Black Mafia. Ethnic Succession in Organized Crime. New York, Simon and Schuster.

Krebs, V. E. (2002). Mapping Networks of Terrorist Cells. Connections, 24(3), 43-54.

Kleemans, E. R. y Van De Bunt, E. G. (1999). Social embeddedness of organized crime. Transnational Organized Crime, 5(1), 19-36.

Kleemans, E.R. y De Poot (2008), Criminal Careers in Organized Crime and Social Opportunity Structure. European Journal of Criminology, 5(1), 6998.

Mclllwain, J.S. (1999). Organized Crime: A social network approach. Crime, Law and Social Change, 32, 301-324.

Monge, P. R. y Contractor, N. (2003). Theories of Communication Network. New York: Oxford University Press.

Morselli, C. (2005). Contacts, opportunities and criminal enterprise. Toronto, Ontario, Canada: University of Toronto Press.

Morselli, C. (2009). Inside Criminal Networks. New York: Springer.

Morselli, C. y Giguère, C. (2006). Legitimate strengths in criminal networks. Crime, Law and Social Change, 45, 185-200. 
Natarajan, M. (2000). Understanding the structure of a drug trafficking organization: A conversational analysis. Crime Prevention Studies, 11, 273-298.

Natarajan, M. (2006). Understanding the structure of a large heroin distribution network: A quantitative analysis of qualitative data. Journal of Quantitative Criminology, 22, 171-192.

Natarajan, M. y Belanger, M. (1998). Varieties of upper-level drug dealing organizations: a typology of cases prosecuted in New York City. Journal of Drug Issues, 28(4), 1005-1026.

Norris, F.H., Stevens, S.P., Pfefferbaum, B., Wyche, K.F., Pfefferbaum, R.L., (2008). Community resilience as a metaphor, theory, set of capacities, and strategy for disaster readiness. American Journal of Community Psychology, 41, 127-150.

Paoli, L. (2002). The Paradoxes of organized crime. Crime, Law \& Social Change, 37, 51-97.

Pearson, G. y Hobbs, D. (2003). Kin pin? A case study of a middle market drug broker. Howard Journal of Criminal Justice, 42(4), 335-347.

Reuter, P.H. (1985). Organization of Illegal Markets: An economic analysis. Washington DC: National Institute of Justice.

Reuter, P. (2004). The political economy of drug smuggling. En M. Vellinga (ed). The political economy of the drug industry. University Press of Florida, Gainesville, 129-147.

Reuter, P.H: y Haaga, J. (1989). The organization of high level drug markets: An exploratory study. Santa Monica, CA: Rand.

Sheffi, Y. (2005). The Resilient Enterprise: Overcoming Vulnerability for Competitive Advantage. Cambridge MA: Massachusetts Institute of Technology.

Sparrow, M. K. (1991). The Application of Network Analysis to Criminal Intelligence. An Assessment of the Prospects. Social Networks, 13, 251274.

Von Lampe, K. (2008). Organized Crime in Europe: Conceptions and Realities. Policing: A Journal of Policy and Practice, 2(1), 7-17.

Walker, B., Holling, C.S., Carpenter, S.R., Kinzig, A. (2004). Resilience, adaptability and transformability in social ecological systems. Ecology and $\begin{array}{lllll}\text { Society } & 9 & (2), & 5 . & \text { (online) }\end{array}$ http://www.ecologyandsociety.org/vol9/iss2/art5/.

Williams, P. (2001). Transnational criminal networks. En Arquilla, J., Ronfeldt, D. (Eds.), Networks and Netwars: The Future of Terror, Crime and Militancy. RAND, Santa Monica, CA, 61-99.

Zaitch, D. (2002). Trafficking cocaine: Colombian drug entrepreneurs in the Netherlands. The Hague, Netherlands: Kluwer.

Zhang, S. y Chin, K. (2003). The declining significance of triad societies in transnational illegal activities. A structural deficiency perspective. British Journal of Criminology, 43(3), 469-488. 